\title{
Superconducting properties of lithographic lead break junctions
}

\author{
David Weber $\odot$ and Elke Scheer ${ }^{1}($ C) \\ Universitätsstr. 10, D 78464 Konstanz, Germany \\ E mail: david.weber@uni konstanz.de and elke.scheer@uni konstanz.de
}

\begin{abstract}
We have fabricated mechanically controlled break junction samples made of lead $(\mathrm{Pb})$ by means of state-of-the-art nanofabrication methods: electron beam lithography and physical vapour deposition. The electrical and magnetic properties were characterized in a ${ }^{3} \mathrm{He}$ cryostat and showed a hard superconducting gap. Temperature and magnetic field dependence of tunnel contacts were compared and quantitatively described by including either thermal broadening of the density of states or pair breaking in the framework of a Skalski model, respectively. We show point contact spectra of few-atom contacts and present tunneling spectra exhibiting a superconducting double-gap structure.
\end{abstract}

Keywords: mechanically controllable break junction, mesoscopic superconductivity, point contact spectroscopy, low temperature, $\mathrm{Pb}$

\section{Introduction}

Due to their well-understood transport properties, atomic-size contacts serve as testbeds for the study of the microscopic origins of a multitude of quantum transport phenomena [1] including, among others, shot noise, thermopower, and superconducting multiparticle transport. The mechanically controlled break junction (MCBJ) technique enables establishing single-atom contacts of a variety of metals, thereby combining tunability and stability over several hours. Of particular interest are nanofabricated MCBJs that lend themselves as electrodes for hybrid junctions in which molecules or nanoparticles are captured [1] and which can be embedded into more complex nanoelectronic circuits [2].

Where superconductivity is concerned, so far the material of choice has been aluminum (Al) due to its robust superconducting properties that are well described by BCS theory and its well-established nanofabrication recipe. A drawback of $\mathrm{Al}$, however, is its relatively low critical temperature of $T_{\mathrm{c}}=1.19 \mathrm{~K}$ that goes along with a rather small gap of $2 \Delta=356 \mu \mathrm{eV}$ and critical field $B_{\mathrm{c}}=10 \mathrm{mT}$. For exploring

${ }^{1}$ Author to whom any correspondence should be addressed the superconducting properties of hybrid devices with $\mathrm{Al}$, measuring at very low temperatures in ${ }^{3} \mathrm{He} /{ }^{4} \mathrm{He}$ dilution refrigerators and sophisticated wiring and filtering are required.

Therefore, other superconductors such as MoRe have been explored to serve as electrode material for molecular junctions using the electromigration technique in a proximity effect arrangement [3, 4]. In these geometries, however, MoRe features a soft gap only and a small coherence length. Furthermore, no MCBJs have been reported so far, because using alloys and compounds render the analysis of transport phenomena on the atomic and single-molecule scale more complex. Thus, an alternative elementary superconductor is needed. In this regard, $\mathrm{Pb}$ appears appealing, because it exhibits $T_{\mathrm{c}}=7.2 \mathrm{~K}$, higher gap energy of $2 \Delta=2700 \mu \mathrm{eV}$ and higher critical field of $B_{\mathrm{c}}=80 \mathrm{mT}$. These properties make studies of subgap transport phenomena more accessible.

$\mathrm{Pb}$ has been used as an electrode material before to create superconducting nanoconstrictions by means of scanning tunneling methods [ 58 , and electromigrated break junctions [9] where it revealed favourable superconducting properties. However, fabricating thin film MCBJs with well-controlled superconducting properties remains challenging because of 
the low melting temperature of $\mathrm{Pb}$ resulting in poor adhesion and granular film growth on many substrates as well as oxidation and contamination issues.

\section{Methods}

\subsection{Sample fabrication}

The MCBJ samples were fabricated by electron beam lithography on flexible substrates. First, a bronze foil (thickness $300 \mu \mathrm{m}$ ) is mechanically polished until the surface roughness is below $1 \mu \mathrm{m}$ rms. As the sacrificial and insulating layer, polyimide (Durimide 115A, Fujifilm Electronic Materials) was used, it is soft baked in an oven at $130{ }^{\circ} \mathrm{C}$ and then cured in high vacuum at $450{ }^{\circ} \mathrm{C}$ for at least $30 \mathrm{~min}$. For electron beam lithography a double-layer resist composed of a buffer layer of MMA-8.5-MAA EL11 (both Micro Chem) and a mask layer of 950k PMMA A4 is spun and hard baked at $170{ }^{\circ} \mathrm{C}$ under atmospheric pressure. The electron beam lithography is performed in a Crossbeam 1540XB system (Zeiss) with Elphy Plus lithography extension (Raith).

After developing in 1:3 MIBK:IPA for $30 \mathrm{~s}$, the samples are mounted in a UHV electron-beam evaporation system. $150180 \mathrm{~nm} \mathrm{~Pb}(5 \mathrm{~N}$, Kurt Lesker Ltd.) is evaporated at $2 \mathrm{~nm} \mathrm{~s}^{-1}$ on the substrate cooled to below $-130^{\circ} \mathrm{C}$ in high vacuum of $1 \times 10^{-7} \mathrm{hPa}$ with subsequent lift-off process in acetone. Final $\mathrm{O}_{2}$ etching of the polyimide with $35 \mathrm{~W}$ RF power (Sentech SI 220) over 20 min establishes a freestanding bridge. The resistance of pristine samples increases typically by $\approx 10 \%-20 \%$ during the dry etching process which we attribute to oxidation of the surface. We can deduct an oxidation of a few $\mathrm{nm}$ from this resistance change. Thus, a film thickness of $>100 \mathrm{~nm}$ is advantageous: it is sufficient to retain a metallic core in the nanobridge as is proven by the temperature dependence of the resistance which reveals metallic behavior with an elastic mean free path corresponding to the grain size observed in the electron micrographs. Since the samples are first broken at low-temperature in cryogenic vacuum, the metallic core is never exposed to a reactive atmosphere and the ex situ preparation does not affect the sample quality.

The indicated fabrication parameter combination is a result of an intensive optimization process. Possible problems when using higher substrate temperatures are partial desorption and large metal grains giving rise to incomplete layer growth. We also tested higher evaporation rates that, however, degrade the mask material hampering a complete mask lift-off, while lower rates increased contamination of the metal film deteriorating the superconducting properties.

\subsection{Measurement setup}

A ${ }^{3} \mathrm{He}$ cryostat (Oxford Instruments Heliox VL) with base temperature of $300 \mathrm{mK}$ and custom-made high-resolution wiring is used [10]. The measurement electronics consists of a real four-wire measurement with voltage and current amplifier including a lock-in amplifier to measure 1st and 2nd

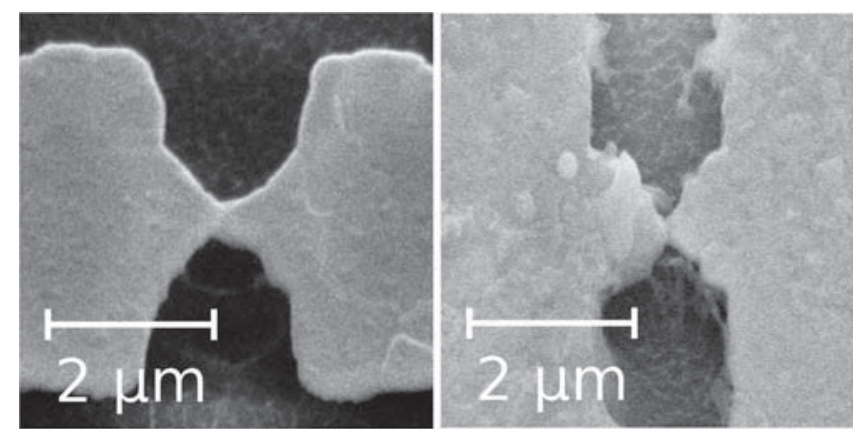

Figure 1. Sample C (left) showing a typical poly crystalline structure with grain sizes in the range of several hundred $\mathrm{nm}$. The width of the bridge is $200 \mathrm{~nm}$ and the layer thickness is $180 \mathrm{~nm}$. Sample E (right) hetero junction with gold $(\mathrm{Au})$ capping layer.

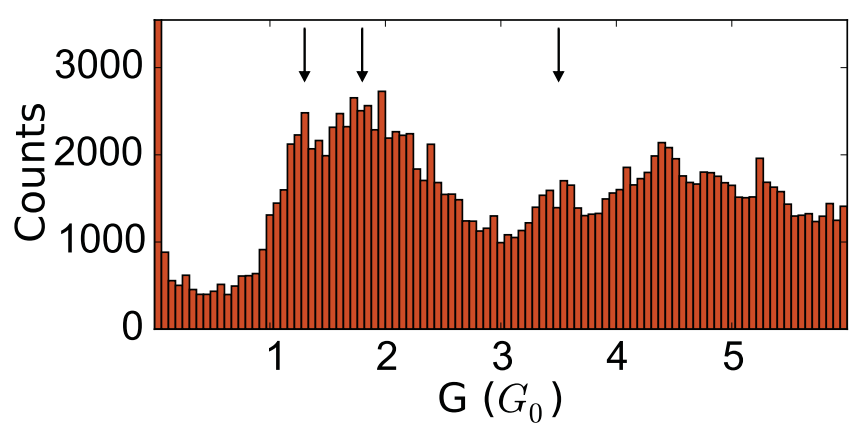

Figure 2. Histogram from 830 traces of sample $\mathrm{B}$ at $8 \mathrm{~K}$ and $0 \mathrm{~T}$ with $20 \mathrm{mV}$ bias applied. Corresponding breaking traces are displayed in the figure $\mathrm{S} 1$.

derivative of voltage $(V)$ and current $(I)$ simultaneously (see figure S2, available online at stacks.iop.org/NANO/29/ 045703/mmedia). A DC motor drives a low-temperature compatible breaking mechanism. The temperature is monitored by a resistive thermometer next to the sample and the magnetic field is applied by a superconducting solenoid system perpendicular to the sample plane.

\section{Results and discussion}

\subsection{Sample properties}

Comparing the samples' resistance values at room and low temperature yields an average residual resistance ratio (RRR) of around 30. With the sample geometry we deduce a mean free path of $l=150-300 \mathrm{~nm} \quad\left(v_{\mathrm{F}}=1.81 \times 10^{8} \mathrm{~cm} \mathrm{~s}^{-1}\right.$, $n=13.2 \times 10^{22} \mathrm{~cm}^{-3}$ ) at low temperature in accordance with observed grain sizes in scanning electron micrographs (figure 1).

Conductance histograms for each sample are calculated from several hundred breaking traces recorded at elevated temperature $\left(8 \mathrm{~K}>T_{\mathrm{c}}\right)$. Examples of breaking curves are shown in the supporting information (SI). An example of a conductance histogram is depicted in figure 2, exhibiting a broad peak around 1.6 and a shallow one at $3.5 \mathrm{G}_{0}$. The positions of the maxima indicate the conductance of preferred atomic configurations of the contacts, while the shape and the 
Table 1. Parameters of four different samples. $T_{\mathrm{c}}$ is the critical temperature deduced from the $R(T)$ curves, $B_{\mathrm{c}}$ is the critical field deferred from the current voltage curves, $\mathrm{RRR}=R_{T_{0}} / R_{0}$ is the residual resistance ratio, where $R_{T_{0}}$ denotes the resistance of the unbroken sample at room temperature and $R_{0}$ the resistance of the unbroken sample at base temperature with a magnetic field $B>B_{\mathrm{c}}$ applied, $\Delta$ is the superconducting gap deduced from tunneling spectra, and $l$ is the inelastic mean free path.

\begin{tabular}{lcccc}
\hline Sample & $\mathrm{A}$ & $\mathrm{B}$ & $\mathrm{C}$ & $\mathrm{D}$ \\
\hline$T_{\mathrm{c}}(\mathrm{K})$ & $7.4 \pm 0.2$ & $7.2 \pm 0.1$ & $7.2 \pm 0.1$ & $7.1 \pm 0.1$ \\
$B_{\mathrm{c}}(\mathrm{mT})$ & $125 \pm 5$ & $125 \pm 5$ & $125 \pm 5$ & $125 \pm 5$ \\
& $@ 0.87 \mathrm{~K}$ & $@ 0.7 \mathrm{~K}$ & $@ 0.45 \mathrm{~K}$ & $@ 0.45 \mathrm{~K}$ \\
$\mathrm{RRR}$ & 36 & 47 & 23 & 17 \\
$R_{T_{0}}(\Omega)$ & 206 & 188 & 83 & 50 \\
$R_{0}(\Omega)$ & 5.8 & 4.0 & 3.55 & 2.9 \\
$\Delta(\mathrm{meV})$ & 1.38 & 1.39 & 1.39 & 1.37 \\
& \pm 0.03 & \pm 0.01 & \pm 0.03 & \pm 0.03 \\
$l(\mathrm{~nm})$ & 160 & 230 & 220 & 270 \\
\hline
\end{tabular}

height of the peaks depend on the experimental realizations [1].

Here, the peaks are somewhat broader and shifted to slightly lower conductance values than reported for STM prepared junctions or MCBJs from bulk wires, as typical for thin-film MCBJs [11 15]. Additionally, a lower number of traces due to the slow operation is recorded.

The first maximum is formed by the last plateaus before breaking and reflects the formation of single-atom contacts, while the second one corresponds to two-atom wide contacts [13]. At variance with earlier observations, the first peak is split into two maxima around 1.3 and $1.8 \mathrm{G}_{0}$. Such a splitting has been observed before for $\mathrm{Zn}$ [16] and Sn [13] with large grain sizes and was attributed to different crystalline orientations of the electrodes. The same effect can be expected for thin-film MCBJs of $\mathrm{Pb}$ as well.

The material specific parameters of four samples are listed in table 1.

Thanks to the outstanding mechanical stability of the lithographically fabricated samples, an established atomicsize contact obtained by stretching or compressing the electrodes is stable over hours. Its lifetime is reduced by sweeping magnetic fields, applying high bias voltages, and by elevated temperatures which will relax the atomic arrangement obtained by mechanical deformation to an energetically more favorable configuration. A deterioration of the sample properties was never experienced within a single cooldown.

\subsection{Tunnel spectra}

Breaking the nanobridge below $T_{\mathrm{c}}$ into the tunneling regime forms a superconductor insulator superconductor junction with typical shape of the current voltage $(I V)$ characteristics with a complete suppression of the current (see inset of figure 3) for $|V|<2 \Delta / e$ which can be used to deduce the superconducting gap parameter $2 \Delta$ of $2750 \pm 50 \mu \mathrm{eV}$ at $450 \mathrm{mK}$, close to the bulk value.

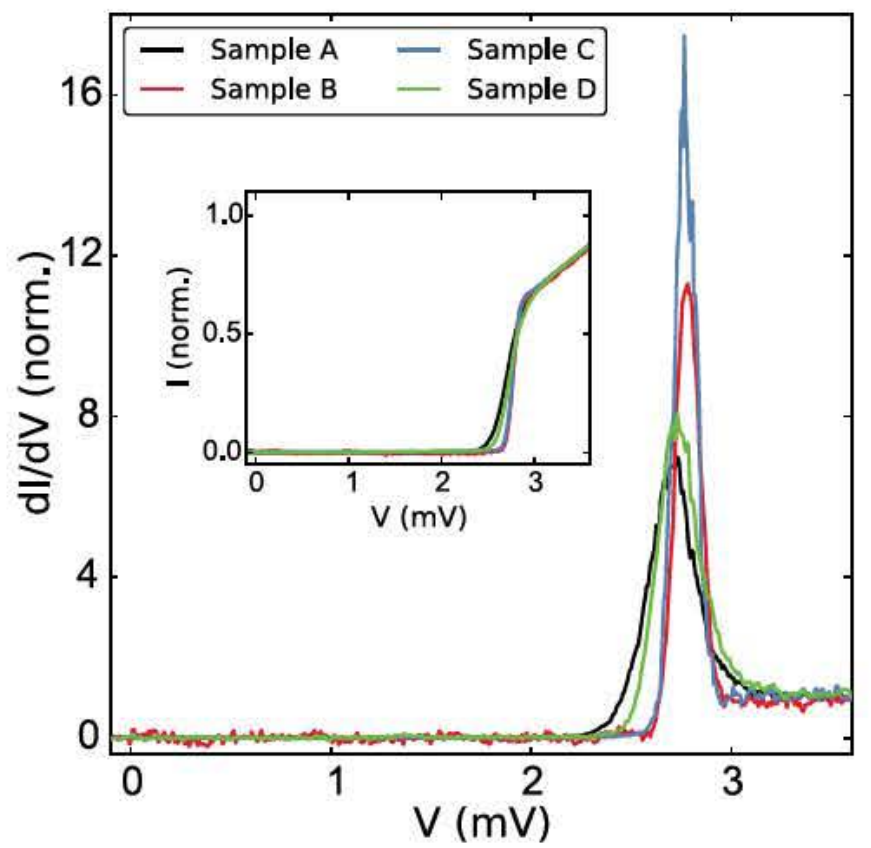

Figure 3. Gap sizes deduced from these plots are summarized in table 1 . The $\mathrm{d} I / \mathrm{d} V$ was calculated by numerical derivation and was normalized to the normal state conductance. The inset shows the corresponding current normalized to give the same current outside the gap $(T<600 \mathrm{mK}, B=0 \mathrm{~T})$.

An increase of the gap has previously been reported for samples with a lower mean free path [17]. The numerical derivative $\mathrm{d} / \mathrm{d} V$ reveals a broadening of the coherence peak at the gap edge on the order of $150 \pm 20 \mu \mathrm{eV}$ full width at half maximum for two samples, while it amounts to $250 \pm 20 \mu \mathrm{eV}$ for the remaining two, presumably because of a small residual magnetic field in the superconducting coil and the high sensitivity of the transport properties to magnetic fields (see below).

We attribute the rounded peaks in the $\mathrm{d} I / \mathrm{d} V$ to a broadening of the density of states (DOS), since the $I V \mathrm{~s}$ in the tunnel regime represent convolutions of the DOS of the electrodes. The transmission probabilities can be assumed to be energy independent in the given small energy range. The measured current is proportional to the integration over the DOS $\rho$, the applied bias voltage $V$ and temperature $T$ with the Fermi function $f$, formulated here with the energy scale $\epsilon$ relative to $E_{\mathrm{F}}$ :

$$
\begin{aligned}
I(V, T) \propto & \int_{-\infty}^{+\infty} \rho(\epsilon) \rho(\epsilon+e V) \\
& \cdot[f(\epsilon, T)-f(\epsilon+e V, T)] \mathrm{d} \epsilon .
\end{aligned}
$$

\subsection{Magnetic field dependence}

The influence of an applied magnetic field on the tunnel spectra is plotted in figure 4 . For the reduced geometry of a mesoscopic contact with lateral dimensions smaller than the London penetration depth the critical field $B_{\mathrm{c}}$ is enhanced, as has been observed before in nanonecks of $\mathrm{Pb}$ formed by the STM technique [7]. In our samples the nonlinearities in the 

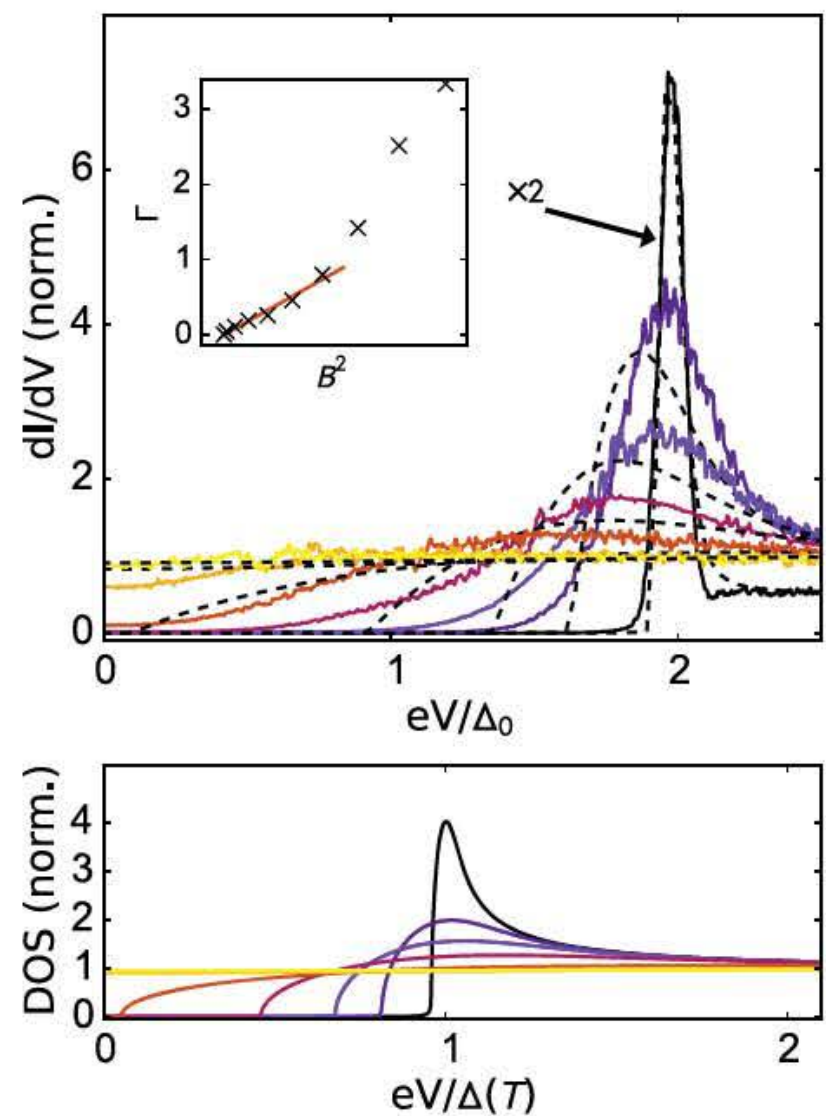

$-B=0 \mathrm{mT}-\mathrm{B}=50 \mathrm{mT}-\mathrm{B}=150 \mathrm{mT}-\mathrm{B}=225 \mathrm{mT}$
$-\mathrm{B}=25 \mathrm{mT}-\mathrm{B}=100 \mathrm{mT}-\mathrm{B}=200 \mathrm{mT}-\mathrm{Fits}$

Figure 4. Sample $\mathrm{C}$ at $500 \mathrm{mK}$. The upper inset shows measured data which was numerically derived (solid line) and a Skalski model fit (dashed) with the according fields. The small inset shows a quadratic dependence of the pair breaking parameter $\Gamma$ as a function of magnetic field with a linear fit over the first seven data points. The lower inset presents the underlying DOS.

$\mathrm{d} I / \mathrm{d} V$ are suppressed at $\approx 200 \mathrm{mT}$. We use a Skalski pair breaking model [18] (see (SI) for the equations) to quantitatively describe the transition from the hard gap in zero field to the normal state. We calculated the $\mathrm{d} I / \mathrm{d} V$ by numerically solving these equations and compared them by the method of least squares to find the best-fitting parameter set. We fit the measured $I V \mathrm{~s}$ assuming a Skalski DOS (see SI3) shown in the bottom panel of figure 4 .

In this model and for the given geometry of a thin film we expect a broadening of the DOS and a transition to gapless superconductivity proportional to the square of the applied field $[19,20]$ which is expected for thin film samples with a parallel field applied:

$$
\Gamma=\frac{D_{\mathrm{el}} e^{2} B^{2} w^{2}}{6 \hbar \Delta} .
$$

Here $D_{\mathrm{el}}=\frac{1}{3} v_{\mathrm{F}} l$ is the electronic diffusion constant with the sample's effective width $w$. Even though we applied a perpendicular field, such a behavior is expected due to the contact geometry, since the voltage drops over the narrowest
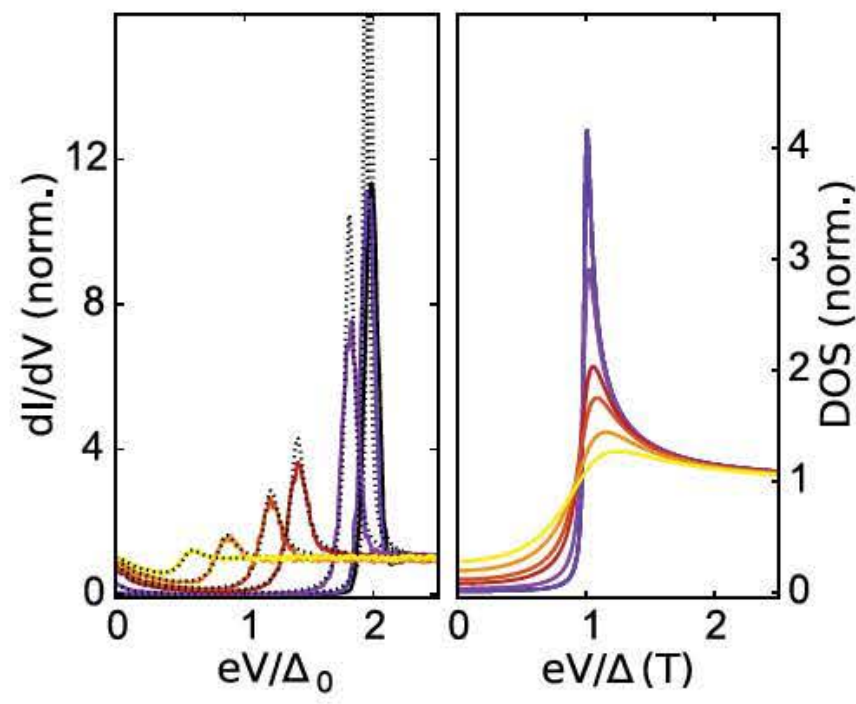

$$
\begin{aligned}
& -1.6 \mathrm{~K}-4.5 \mathrm{~K}-6.5 \mathrm{~K}-7.2 \mathrm{~K} \\
& -3.0 \mathrm{~K}-6.0 \mathrm{~K}-7.0 \mathrm{~K} \cdots . . . \mathrm{Fits} \\
& \hline
\end{aligned}
$$

Figure 5. Fitting with a phenomenological Lorentzian distribution of $\Delta(T)$ for sample $\mathrm{C}$ in zero magnetic field. A Lorentzian broadening over this DOS was combined with a Fermi function with temperature $T_{\text {fit }}$ for the current integration. The left plot shows the numerical derivative of the measured data (solid) with fits (dotted). The right plot shows the DOS with a Lorentzian convolution. Corresponding fitting parameters are shown in figure 6.

Table 2. Fitting results for the Skalski model of the magnetic field dependent data shown in figure 4.

\begin{tabular}{llcc}
\hline Field $(\mathrm{mT})$ & $\Gamma$ & Field $(\mathrm{mT})$ & $\Gamma$ \\
\hline 0 & 0.006 & 125 & 0.51 \\
25 & 0.04 & 150 & 0.83 \\
50 & 0.1 & 175 & 1.4 \\
75 & 0.18 & 200 & 2.9 \\
100 & 0.3 & 225 & 3.8 \\
\hline
\end{tabular}

part where the sample forms a wire with approximately square-shaped cross-section $[7,21]$.

The initial broadening in zero field is accounted for by a small $\Gamma=6 \times 10^{-3}$. The resulting pair breaking parameters $\Gamma$ are plotted in the inset as a function of $B^{2}$ and listed in table 2 . The roughly linear dependence at small fields supports the validity of the model. For higher fields the precision with which $\Gamma$ can be determined is limited and the fitting parameter range was cut off at 3.8 .

\subsection{Temperature dependent DOS broadening}

Examples of tunnel spectra for sample $\mathrm{C}$ at various temperatures are plotted in figure 5. Beside a reduction of the gap $\Delta \rightarrow \Delta(T)$ when approaching $T_{\mathrm{c}}$, as expected, we observe a pronounced broadening of the coherence peak that goes beyond mere thermal broadening of the Fermi functions, as a tentative fitting reveals (see figure $\mathrm{S} 3$ ).

This observation is distinctly different from the behavior of $\mathrm{Al}$ where only negligible broadening was observed [21]. 


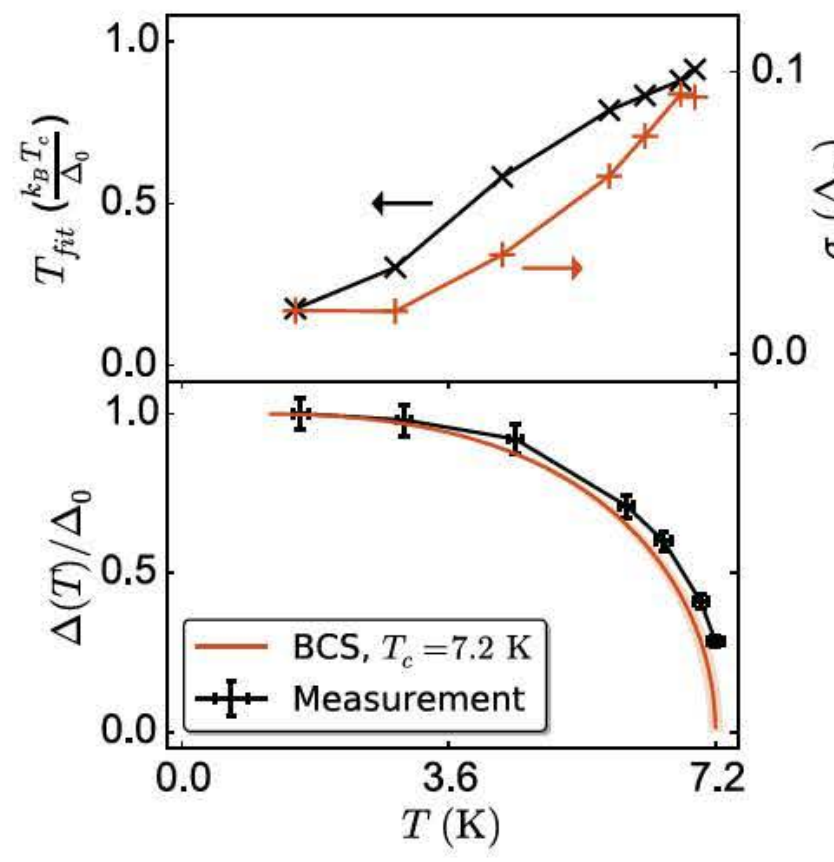

Figure 6. The upper plot shows the dependence of the fitting parameters on the sample temperature using a Lorentzian broad ening. The fit exhibits mainly a linear trend for the Fermi function fit temperature $T_{\text {fit }}$. It shows a monotonic, but super linear trend for the broadening parameter with temperature. The numerically calculated fitting errors are negligibly small. The gap size was adjusted individually at each temperature and shows a monotonous decay to $T_{\mathrm{c}}(\Delta(T \approx 0) \approx 1.40 \mathrm{meV})$ in the lower plot. The prediction of the BCS theory for weak coupling superconductors is shown in red.

However, the finite DOS in the gap $(V<2 \Delta(T) / e)$ can be well described by the Fermi function with temperature $T_{\text {fit }}$.

To account for the additional rounding of the coherence peaks, we included an empirical broadening parameter $\sigma(T)$ by convoluting a slightly broadened Skalski DOS $\left(\Gamma=1 \times 10^{-3}\right)$ with a Lorentzian distribution $L$ when calculating the differential conductance:

$$
L(\epsilon, \sigma)=\frac{1}{\pi} \frac{\sigma}{\sigma^{2}+\epsilon^{2}} .
$$

The latter is motivated by the fact that $\mathrm{Pb}$ is known to be a two-band superconductor with differing gap size, where the relative weight of appearance of the gaps in the DOS depends on the crystal orientation [22]. Here we deal with polycrystalline samples with arbitrary orientation of the grains and can thus expect a smearing of the DOS at the gap edge. The results of the Lorentzian broadening model is displayed in figure 5. $T_{\text {fit }}$ and broadening $\sigma(T)$ are plotted as a function of temperature in the overview, see figure 6.

The fitting with the Lorentzian distribution of gaps describes the experimental data well. Two other models to describe the observed temperature dependence of the spectra are presented in the SI. The width of the Lorentzian curve as a function of temperature follows a linear behavior. The distribution of gaps at zero temperature is expected to result also in a variation of the temperature dependence of the gap.

The $\Delta(T)$ values are adjusted individually because $\mathrm{Pb}$ is a strong coupling superconductor for which the absolute gap

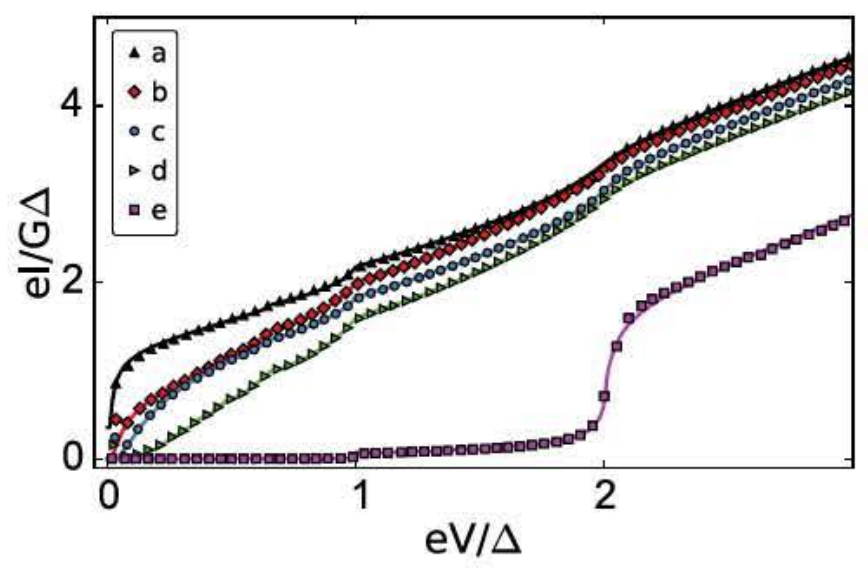

Figure 7. The figure shows measurements (symbols) and fits (lines) of five different contacts recorded on sample $\mathrm{C}$ at $T=350 \mathrm{mK}$ and $B=0 \mathrm{~T}$ with $\Delta=1360 \mu \mathrm{eV}$. The total conductance and the transmissions $\tau_{i},(i=0,1, \ldots)$ of the five contacts were determined as follows: (a) $1.77 \mathrm{G}_{0}: \tau_{0}=1.00, \tau_{1}=0.41, \tau_{2}=0.36$ (b) $2.55 \mathrm{G}_{0}$ : $\tau_{0}=0.98, \tau_{1}=0.75, \tau_{2}=0.41, \tau_{3}=0.40$ (c) $1.82 \mathrm{G}_{0}: \tau_{0}=0.95$, $\tau_{1}=0.48, \tau_{2}=0.22, \tau_{3}=0.17$ (d) $1.56 \mathrm{G}_{0}: \tau_{0}=0.85, \tau_{1}=0.51$, $\tau_{2}=0.20$ (e) $0.09 \mathrm{G}_{0}: \tau_{0}=0.08, \tau_{1}=0.01$.

size as well as the temperature dependence of the gap differs from the generic BCS behavior. The evolution of $\Delta(T)$ is plotted in the lower part of figure 6.

\subsection{Multiple Andreev reflections (MAR)}

When closing the MCBJ to form atomic contacts, nonlinearities in the $I \mathrm{Vs}$ occur at subgap voltages. These arise from MAR [23, 24], and the exact shape of the $I V \mathrm{~s}$ depends on the number and transmission probabilities of the conduction channels of the atomic contact [11], enabling to correlate the transport properties to atomic configurations [25]. Examples of $I V \mathrm{~s}$ recorded on contacts with conductances in the first histogram maximum and the tunnel regime of sample $\mathrm{C}$ are plotted in figure 7. The experimental data is compared to a theoretical model of MAR [23] to determine the number of conduction channels in the contact. The fitted curves together with the best-fit parameters are also shown in figure 7.

The quality of the fits is comparable to the ones obtained before from STM break junctions of $\mathrm{Pb}[5,7,11]$. Due to the finite measurement temperature the number of channels that can be determined precisely is estimated to be seven. When performing the procedure at higher temperatures, the thermal broadening reduces the resolution, resulting in a smaller maximum number of unambiguously disentangled channels.

For contacts with conductances between 1.5 and $2.6 \mathrm{G}_{0}$, we find 3 to 4 conduction channels as expected for singleatom contacts of $\mathrm{Pb}[11]$ and observed before in STM-based experiments [26]. This finding supports the interpretation of the first histogram peak to represent single-atom contacts. The transmission values are in general not quantized, but often one dominating channel achieves transmission values only slightly below $\tau_{i}=1$, as found earlier for single-atom $\mathrm{Pb}$ contacts $[5,7,11]$. 


\subsection{Phonon spectra}

$\mathrm{Pb}$ as a soft metal reveals van Hove singularities in the phonon DOS at roughly $4 \mathrm{meV}$ (TA phonons) and $8.6 \mathrm{meV}$ (LA phonon), just slightly above the superconducting gap [27]. These reveal themselves as minima in the point contact spectra (PCS), $\mathrm{d}^{2} I / \mathrm{d} V^{2}$, of junctions with several nm diameter fabricated by the classical needle-anvil method [28]. When reducing the size of the contacts to the atomic scale, a variation of the phonon energies and the appearance of soft phonons has been predicted [29]. Furthermore, when breaking the contacts to form tunnel contacts, a transition to inelastic electron tunneling spectroscopy (IETS) is expected [30]. In the single-channel case for transmissions larger than 0.5 , the phonon excitations are expected to appear as minima in the $\mathrm{d}^{2} I / \mathrm{d} V^{2}$ for positive bias. For smaller transmissions, phonon excitation is signaled by maxima in positive bias, corresponding to the IETS case. Since the spectra are expected to be antisymmetric with respect to bias reversal, the sign change holds vice versa for negative bias [31]. Deviations from the antisymmetry indicate that additional features such as conductance fluctuations contribute to the transport, as has been observed before in, for example, $\mathrm{Au}$ [32] or Pd [33] atomic contacts.

To test these predictions, we study the second derivative of the $I \mathrm{Vs}$ of atomic contacts made with the lithographic MCBJ technique at $T=650 \mathrm{mK}$ and $B=1 \mathrm{~T}$ in the normal conducting state. The outstanding stability of this technique enables us to take consecutive measurements over hours. Figure 8 shows a set of PCS recorded when slowly stretching a contact from $4 \mathrm{G}_{0}$ to $0.46 \mathrm{G}_{0}$. For all spectra we observe a pronounced minimum maximum combination around zero bias. This shape indicates a minimum of the differential conductance around zero bias (see figure S5). This zero-bias anomaly (ZBA) is interpreted to arise from dynamic Coulomb blockade (DCB). DCB is expected to occur due to the reduced screening of the conduction electrons in the narrow constriction [34]. The observability of DCB depends on the frequency-dependent impedance of the circuit in which the junction is embedded. It was observed in $\mathrm{Al}$ atomic contacts when adding on purpose resistive leads before [34]. $\mathrm{Pb}$ has a relatively large normal state resistivity sufficient to observe remainders of $\mathrm{DCB}$ without additional lead resistance.

Besides the DCB structure we observe additional features in the voltage range of $\pm 10 \mathrm{mV}$, the shape of which varies from contact to contact and which we will discuss now, starting with the largest contacts. By comparison with the conductance histogram, the contacts with $G>4 \mathrm{G}_{0}$ correspond to contacts with more than two atoms in cross-section. In addition to the $\mathrm{ZBA}$, the spectrum of the thickest contact with $G=4.6 \mathrm{G}_{0}$ reveals shallow maxima at $-9(\Delta)$ and $-5 \mathrm{mV}(\boldsymbol{\square})$ and weak minima at $+5(\boldsymbol{\square})$ and $+9 \mathrm{mV}(\boldsymbol{\Delta})$. These features appear close to the bulk phonon energies and are therefore tentatively attributed to phonon scattering. They remain visible for contacts with $G \approx 3 \mathrm{G}_{0}$, which represent two-atom contacts.

When further stretching the junction, the structures at $\pm 9 \mathrm{mV}$ fade out, move to somewhat smaller $|V|$ value and

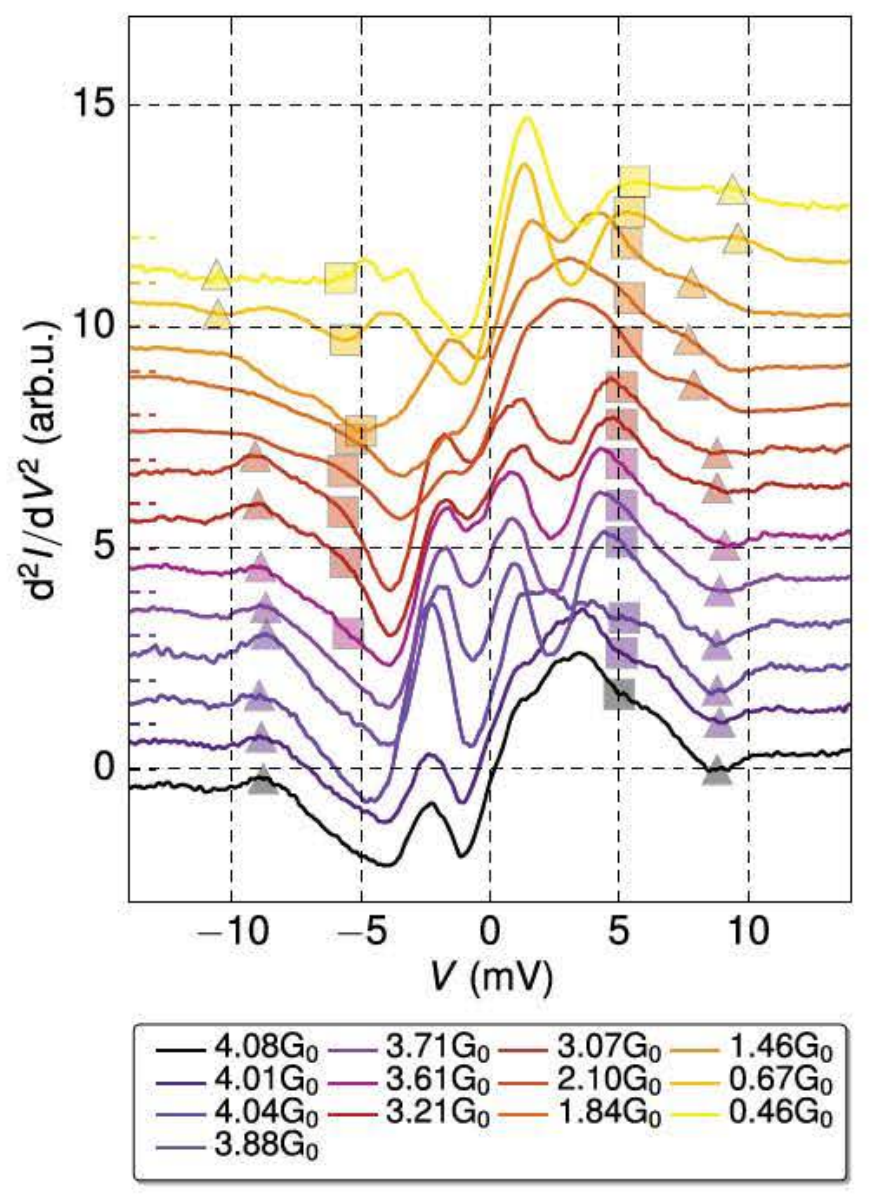

Figure 8. Set of consecutive PC spectra (offset vertically) of a $\mathrm{Pb}$ contact while slowly breaking (from bottom to top) at $T=650 \mathrm{mK}$ and $B=1 \mathrm{~T}$. The evolution of the two $\mathrm{Pb}$ phonon modes are highlighted by $\boldsymbol{\Lambda}$ and $\mathbf{E}$. Intermediate curves showing almost the same shape are hidden for clarity. The corresponding conductance measurements are shown in figure S5.

eventually invert sign. The structure at $+5 \mathrm{mV}$ also inverts its character upon stretching, while the feature at $-5 \mathrm{mV}$ is difficult to follow because of its vicinity to the ZBA. Nevertheless the gross behavior follows the expected transition from the PCS behavior of large contacts to the IETS behavior for tunneling. In the present case, the transition seems to occur around $G \approx 2 \mathrm{G}_{0}$, i.e. when approaching the single atom regime. In view of the MAR analysis in which still a very well transmitting channel with $\tau>0.5$ was found also for the single-atom contacts, we conclude that the phonons are excited by one of less transmitted channels. Finally, in the tunnel regime (contacts with $0.46 \mathrm{G}_{0}$ and $0.67 \mathrm{G}_{0}$ ) two distinct maxima in the positive and a minimum in the negative bias branch are observed, as expected for the IETS case with all channel transmissions $<0.5$.

Summarizing, the two limiting cases correspond well to the known PCS and IETS behavior, while when the contacts are disordered, the phonon signal is masked by conductance fluctuations which also change their appearance upon reconfiguration of the contact, thus hampering an unambiguous assignment of all features to particular physical effect. Still, the possibility to fine-tune the contact size with sub-atomic 


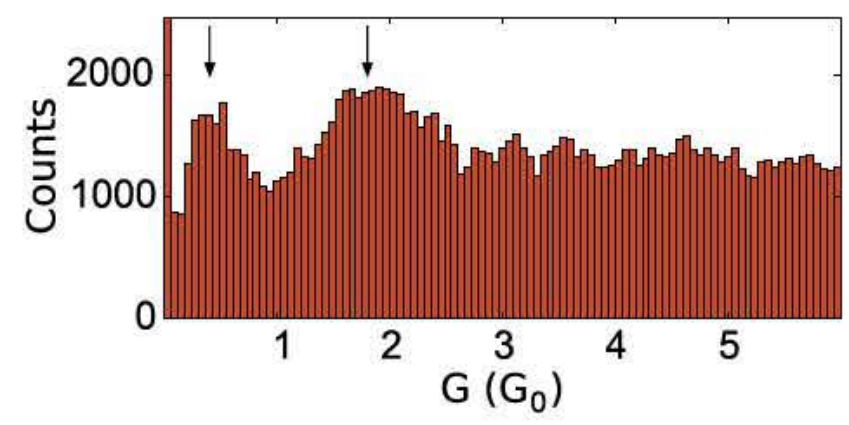

Figure 9. Conductance histogram of sample $\mathrm{E}(150 \mathrm{~nm} \mathrm{~Pb}$ at $10 \AA \mathrm{s}^{-1}, 20 \mathrm{~nm} \mathrm{Au}$ at $1 \AA \mathrm{s}^{-1}$ in situ, both at $25^{\circ} \mathrm{C}$ ) with 623 breaking traces $(20 \mathrm{mV}, 5 \mathrm{~K}, 0 \mathrm{~T})$.

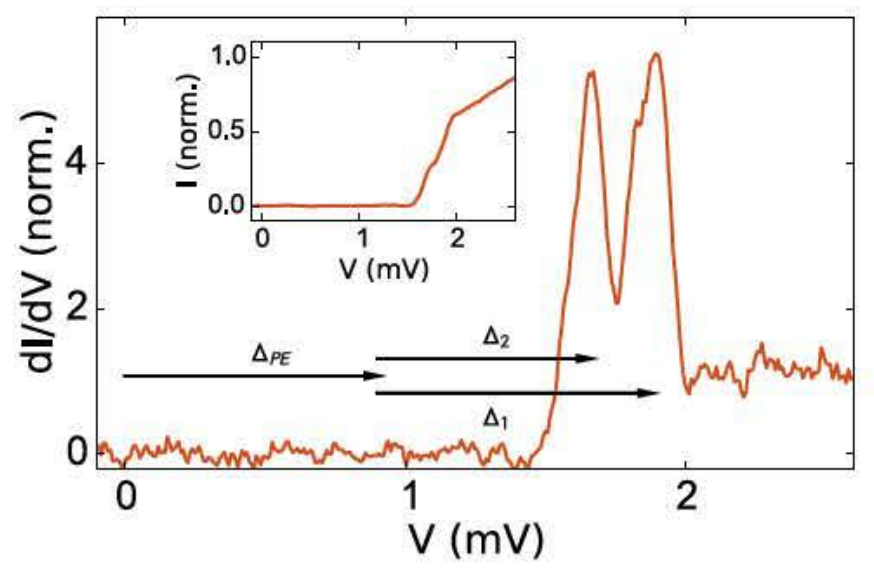

Figure 10. Sample E shows the well defined tunneling gap with $\Delta_{\mathrm{PE}} \approx 900 \mu \mathrm{eV}$ and a peak separation of $230 \mu \mathrm{eV}$ in $\mathrm{d} I / \mathrm{d} V$ at 600 $\mathrm{mK}$ and zero magnetic field. The reduced gap size is attributed to negative proximity effect induced by the protecting Au layer.

precision opens the possibility to optimize the sensitivity to the targeted physical phenomenon.

\subsection{Resolving the double gap of $\mathrm{Pb}$}

$\mathrm{As} \mathrm{Pb}$ is already known to show more than one distinct gap energy [35 37], a recent STM study revealed a crystaldirection dependent gap size in $\mathrm{Pb}$ tunnel junctions, confirming $\mathrm{Pb}$ to be a two-band superconductor. The energy difference between the smallest and largest gap amounted to around $150 \mu \mathrm{eV}$ [22]. As mentioned before, we attribute the finite width of the coherence peaks of roughly $150 \mu \mathrm{eV}$, observed in our polycrystalline junctions, to arise from averaging over different gaps sizes. To force a particular growth direction with the aim to favor a particular gap size, we fabricated a sample consisting of $150 \mathrm{~nm} \mathrm{~Pb}$ covered by a $20 \mathrm{~nm}$ thick Au layer (see figure 1, right). The conductance histogram presented in figure 9 shows no peak around $1 \mathrm{G}_{0}$ (as would be expected for Au atomic contacts [1]), but two broad peaks around 0.4 and $1.7 \mathrm{G}_{0}$. While the origin of the peak around $0.4 \mathrm{G}_{0}$ is unclear, the latter one corresponds well to the observations for the pure $\mathrm{Pb}$ samples. When breaking the sample to form a tunnel contact, we observe a double step of the $I V$ at the gap edge around $2 \Delta_{\mathrm{PE}} \approx 1800 \mu \mathrm{eV}$, see inset of figure 10. The differential conductance depicted in the main plot reveals two peaks offset by $230 \mu \mathrm{eV}$. The overall reduction of the gap is attributed to the negative proximity effect. The splitting is, however, bigger than in the clean $\mathrm{Pb}$ case [22]. But $a b$ initio calculations expect higher splitting [38] and some earlier results present a variety of gap energies $[35,36]$. Since the larger gap in pure $\mathrm{Pb}$ is given by electrons originating from the $\mathrm{p}-\mathrm{d}$ band and the smaller gap by $\mathrm{s}-\mathrm{p}$ electrons, we conclude that the s-p band superconductivity is more affected by the negative proximity effect than the p-d system, thus enhancing the apparent splitting. This interpretation is in accordance with the fact that the charge carriers in the $\mathrm{Au}$ are also s-electrons, their hybridization with the s-p electrons from the $\mathrm{Pb}$ can be expected to be more pronounced.

\section{Conclusion}

In conclusion we presented a fabrication scheme and thorough characterization of tunable superconducting tunnel and few-atom contacts made of thin-film break junctions of $\mathrm{Pb}$. The superconducting properties are close to the ones of bulk $\mathrm{Pb}$, except for an enhanced critical field due to the small lateral size. In the contact regime the subharmonic gap structure caused by MAR is well-developed and enables the determination of the number and transmission coefficients of the Landauer conduction channels. Besides $\mathrm{Al}$ and $\mathrm{Nb}, \mathrm{Pb}$ is only the third elementary superconductor to combine a BCSlike spectrum with nano-fabricated break junctions [39]. When proximitized with $\mathrm{Au}$ the double-band superconductivity of $\mathrm{Pb}$ is resolved. The higher critical temperature, larger gap size and higher critical field of $\mathrm{Pb}$ compared to $\mathrm{Al}$ make $\mathrm{Pb}$ lithographic break junctions suitable as leads for contacting nano-objects like molecules, clusters or quantum dots and to reveal their quantum transport properties.

\section{Acknowledgments}

We thank F Pauly, K J Franke, and H S J van der Zant for helpful discussion, M Ruby and $\mathrm{K} J$ Franke for sharing their codes [40], E Leary for careful proof reading, and S Haus for help in sample fabrication. We acknowledge financial support by the German Research Foundation (DFG) for funding through SFB $767 \mathrm{C} 2$ and SCHE 505/12 and by the Baden-Württemberg Stiftung through the Research Network 'Functional Nanostructures'.

\section{ORCID iDs}

David Weber $₫$ https://orcid.org/0000-0002-2995-1980 Elke Scheer $\oplus$ https://orcid.org/0000-0003-3788-6979 


\section{References}

[1] Agraït N, Levy Yeyati A and van Ruitenbeek J M 2003 Phys. Rep. 37781279

[2] Goffman M F, Cron R, Levy Yeyati A, Joyez P, Devoret M H, Esteve D and Urbina C 2000 Phys. Rev. Lett. 851703

[3] Gaudenzi R, Island J O, de Bruijckere J, Burzurí E, Klapwijk T M and van der Zant H S J 2015 Appl. Phys. Lett. 106222602

[4] Island J O et al 2017 Phys. Rev. Lett. 118117001

[5] Suderow H, Bascones E, Belzig W, Guinea F and Vieira S 2000 Europhys. Lett. 50749

[6] Suderow H, Bascones E, Izquierdo A, Guinea F and Vieira S 2002 Phys. Rev. B 65100519

[7] Rodrigo J G, Suderow H, Vieira S, Bascones E and Guinea F 2004 J. Phys.: Condens. Matter 16 R1151

[8] Franke K J, Schulze G and Pascual J I 2011 Science 3329404

[9] Luo K and Yao Z 2009 Appl. Phys. Lett. 95113115

[10] Schirm C, Pernau H F and Scheer E 2009 Rev. Sci. Instrum. 80 024704

[11] Scheer E, Agraï N, Cuevas J C, Levy Yeyati A, Ludoph B, Martín Rodero A, Bollinger G R, van Ruitenbeek J M and Urbina C 1998 Nature 3941547

[12] Xie F Q, Hüser F, Pauly F, Obermair C, Schön G and Schimmel T 2010 Phys. Rev. B 82075417

[13] Smit R H M 2003 From Quantum Point Contacts to Monatomic Chains: Fabrication and Characterization of the Ultimate Nanowire (Leiden: Universiteit Leiden)

[14] Makk P, Csonka S and Halbritter A 2008 Phys. Rev. B 78 045414

[15] Yanson A I 2001 Atomic Chains and Electronic Shells: Quantum Mechanisms for the Formation of Nanowires (Leiden: Leiden University)

[16] Scheer E, Konrad P, Bacca C, Mayer Gindner A, v Löhneysen H, Häfner M and Cuevas J C 2006 Phys. Rev. B 74205430

[17] Ziemba G and Bergmann G 1970 Z. Phys. 2374108

[18] Skalski S, Betbeder Matibet O and Weiss P R 1964 Phys. Rev. 136 A1500 18

[19] Belzig W, Bruder C and Schön G 1996 Phys. Rev. B 54 94438

[20] Tinkham M 2004 Introduction to Superconductivity (Dover Books on Physics vol 1) 2nd edn (New York: Dover)

[21] Scheer E, Cuevas J C, Levy Yeyati A, Martín Rodero A Joyez P, Devoret M H, Esteve D and Urbina C 2000 Physica B 28042531
[22] Ruby M, Heinrich B W, Pascual J I and Franke K J 2015 Phys. Rev. Lett. 114157001

[23] Cuevas J C, Martín Rodero A and Levy Yeyati A 1996 Phys. Rev. B 547366

[24] Johansson G, Bratus E N, Shumeiko V S and Wendin G 1999 Phys. Rev. B 60138293

[25] Schirm C, Matt M, Pauly F, Cuevas J C, Nielaba P and Scheer E 2013 Nat. Nanotechnol. 86458

[26] Rubio Bollinger G, de las Heras C, Bascones E, Agraït N, Guinea F and Vieira S 2003 Phys. Rev. B 67121407

[27] Schober H R and Dederichs P H 1981 Electron states and fermi surfaces of alloys $\cdot \mathrm{Pb}$ Phonon States of Elements. Electron States and Fermi Surfaces of Alloys (Landolt Börnstein Group III Condensed Matter vol 13A) (Berlin: Springer)

[28] Naidyuk Y and Yanson I 2005 Point Contact Spectroscopy (Springer Series in Solid State Sciences vol 145) 1st edn (New York: Springer)

[29] Bürkle M, Böhler T, Viljas J, Edtbauer A, Weigend F, Schön G, Scheer E and Pauly F 2013 Electron vibration interactions in charge transport through metallic single atom contacts Advances in Nanotechnology (Stuttgart: Baden Württemberg Stiftung)

[30] Cuevas J and Scheer E 2017 Molecular Electronics: An Introduction to Theory and Experiment (World Scientific Series in Nanoscience and Nanotechnology) (Singapore: World Scientific)

[31] Paulsson M, Frederiksen T, Ueba H, Lorente N and Brandbyge M 2008 Phys. Rev. Lett. 100226604

[32] Böhler T, Edtbauer A and Scheer E 2007 Phys. Rev. B 76 125432

[33] Strigl F, Keller M, Weber D, Pietsch T and Scheer E 2016 Phys. Rev. B 94144431

[34] Con R, Yeyati A L, Martin Rodero A, Joyez P, Devoret M, Esteve D, Vecino E and Urbina C 2001 Dynamical Coulomb blockade in quantum point contacts 36th Rencontres de Moriond: Workshop on Electronic Correlations (Les Arcs: EDP Sciences) Moriond workshops

[35] Blackford B L and March R H 1969 Phys. Rev. 1863979

[36] Lykken G I, Geiger A L, Dy K S and Mitchell E N 1971 Phys. Rev. B 4152330

[37] Townsend P and Sutton J 1962 Phys. Rev. 1285915

[38] Floris A, Sanna A, Massidda S and Gross E K U 2007 Phys. Rev. B 75054508

[39] Dai Z, Zhang C, Barnett R N, Marchenkov A and Landman U 2007 Phys. Status Solidi a 204171220

[40] Ruby M 2016 SoftwareX 5316 